\title{
Concepções de Profissionais de Saúde sobre as Atribuições de um Centro de Atenção Psicossocial Infanto-Juvenil
}

\author{
Meyrielle Belotti*, Camila Carlos Maia, Luziane Zacché Avellar, \\ $\&$ Priscilla de Oliveira Martins da Silva \\ Universidade Federal do Espírito Santo, Vitória, ES, Brasil
}

\begin{abstract}
RESUMO - O objetivo do artigo é analisar os significados conferidos pelos profissionais de saúde sobre as atribuições de um Centro de Atenção Psicossocial Infanto-juvenil (CAPSi) de uma Região do Sudeste do Brasil. Utilizou-se a abordagem qualitativa. Participaram do estudo 15 profissionais. Para a coleta de dados, foi utilizada a técnica da entrevista semiestruturada e os dados foram submetidos à análise de conteúdo. Os resultados indicam que as atribuições nomeadas pelos profissionais, em sua maioria, estão em consonância com a proposta do Ministério da Saúde. O estudo também mostra a importância de compreender melhor as atribuições do CAPSi diante da implantação da Rede de Atenção Psicossocial (RAPS). Observou-se a dificuldade dos entrevistados em contemplar as particularidades do cuidado em saúde mental infanto-juvenil.
\end{abstract}

PALAVRAS-CHAVE: serviços de saúde mental, criança, adolescentes

\section{Health Professionals' Conceptions About the Duties of a Children and Adolescents Psychosocial Care Center}

\begin{abstract}
The aim of this paper was to analyze the meanings conferred by health professionals to the duties of a Children and Adolescents Psychosocial Care Center (CAPSi) in a southeastern region of Brazil. A qualitative approach was used. Study participants consisted of 15 professionals. For data collection, we used the technique of semistructured interviews and data were subjected to content analysis. The results indicate that the duties listed by the professionals are mostly in line with the proposal of the Ministry of Health. The study also shows the importance of better understanding the CAPSi duties regarding the implementation of the Psychosocial Care Network (RAPS). There was a difficulty of respondents to consider the care peculiarities in children and adolescents' mental health.
\end{abstract}

KEYWORDS: mental health services, children, adolescents

A inclusão da temática da saúde mental infanto-juvenil nas políticas públicas da saúde mental ocorreu de forma tardia, sendo essa constituída pela extensão das práticas voltadas para a população adulta (Couto, Duarte, \& Delgado, 2008). Esse cenário não se restringe ao Brasil. Em diferentes países, é notória a defasagem entre a necessidade de atenção em saúde mental infanto-juvenil e a oferta de uma rede de cuidados capaz de responder às suas demandas (Pate et al., 2007).

De acordo com a Organização Panamericana de Saúde (OPAS) e a Organização Mundial de Saúde (OMS), até o ano de 2001, 90\% dos países não possuíam políticas públicas de saúde mental que incluíssem crianças e adolescentes. A
OMS aponta alguns motivos para justificar esse cenário, tais como: a falta de divulgação de conhecimento sobre o desenvolvimento e os sofrimentos psíquicos da criança e do adolescente, a ausência de capacitação profissional e a escassez de recursos financeiros (OMS, 2005). Assim, historicamente, sabe-se que existe uma lacuna na efetivação das políticas públicas para crianças e para adolescentes. Essa lacuna favoreceu a criação e o fortalecimento de instituições totais de natureza privada e filantrópica que, em sua maioria, atuam de forma fragmentada (Brasil, 2005b).

Para além dessa realidade, cabe ressaltar que a complexidade que permeia o cuidado em saúde mental infanto-juvenil não consegue ser contemplada pelas políticas

\footnotetext{
*E-mail: meyri.belotti@gmail.com
} 
vigentes no Brasil (Couto et al., 2008). A Política de Saúde Mental que hoje se implementa no Brasil é um esforço no sentido de vencer a lacuna assistencial histórica nesse campo (Amstalden, Hoffmann, \& Monteiro, 2010). Diante desse cenário, o presente estudo pretende contribuir para uma discussão significativa sobre as atribuições do CAPSi, bem como para a ampliação do conhecimento sobre as particularidades das estratégias de cuidado no CAPSi que ainda estão em processo de construção. Para isso, seu objetivo consiste em compreender os significados conferidos pelos profissionais de saúde sobre as atribuições de um Centro de Atenção Psicossocial Infanto-juvenil (CAPSi).

Como já relatado em diversos estudos (Amarante, 1995, Birman \& Costa, 1994; Gonçalves \& Sena, 2001), o Movimento da Reforma Psiquiátrica Brasileira, iniciado no fim da década de 1970, possibilitou a implantação de um novo modelo de atenção à saúde mental, denominado atenção psicossocial. Esse modelo compreende o processo de saúde-doença enquanto um fenômeno social complexo e que, por isso, necessita de uma articulação intersetorial e uma abordagem interdisciplinar, bem como a construção de dispositivos territorializados e substitutivos à lógica manicomial. Suas estratégias de cuidado têm, como pressuposto, o acompanhamento dos sujeitos com sofrimento psíquico no seu meio social (território, família, comunidade, entre outros), visando a retomada de autonomia, o resgate da cidadania, a abordagem mais ampla do cuidado e a reinserção social (Yasui \& Costa-Rosa, 2008; Pinto et al., 2011).

Entende-se, neste estudo, a reinserção social como a criação ou o fortalecimento de uma rede de apoio comprometida ou inexistente, tendo como objetivo proporcionar aos sujeitos a plenitude dos seus direitos civis, sendo esse um processo longo, gradativo e dinâmico, pois implica em uma revisão dos estigmas instituídos (Observatório Brasileiro de Informações sobre Drogas [OBID], 2007).

A relação da sociedade com o sujeito estigmatizado pode ocorrer por múltiplas vias, variando entre dois extremos: a superproteção e a rejeição. Ambas as formas resultam no impedimento do desenvolvimento de uma vida em sociedade. Na vivência da saúde mental, frequentemente verifica-se que a sociedade, de uma maneira geral, avalia os sujeitos com sofrimento psíquico por meio de sentimentos de periculosidade e de incapacidade. Esses sentimentos se caracterizam pela via da rejeição, sendo essa uma das principais barreiras para a efetivação das práticas de reinserção social (Jorge et al., 2003).

Cabe destacar que as discussões sobre a reinserção social no campo da saúde mental focam, principalmente, nas questões vinculadas ao trabalho e à geração de renda (Leão \& Barros, 2011). No entanto, quando esse debate é ampliado para a saúde mental infanto-juvenil, essas questões muitas vezes não se enquadram, pois, nessa etapa de vida, geralmente ainda não há a inserção no mercado de trabalho, exceto na etapa final da adolescência, em que já é possível pensar nessas ações, respeitando o que é preconizado no Estatuto da Criança e do Adolescente (ECA).

No que se refere à organização dos serviços de saúde mental infanto-juvenis, de acordo com o Ministério da Saúde (2013), esses devem ser estruturados a partir de uma rede de cuidados que considera as singularidades e as especificidades da criança e do adolescente. Assim, os componentes da rede assumem uma função social que extrapola o fazer técnico do tratar, incluindo ações de acolher, escutar, cuidar, possibilitar ações emancipatórias, enfrentar estigmas e determinismos e promover a qualidade de vida.

Couto e Delgado (2015) enfatizam a necessidade de substanciais investimentos pelas esferas de gestão do SUS, para que possam se inscrever na realidade brasileira como marco de um novo tempo e de um novo modo de cuidar e tratar de crianças e adolescentes em sofrimento mental. Nesse cenário, as estratégias de cuidado devem ser pensadas de forma a contemplar as necessidades: de incluir a família; de adequar a linguagem; de inserir o lúdico no campo das intervenções e de incluir outros setores nas discussões, como: saúde geral, pedagógicos e judiciários (Avellar \& Bertollo, 2008; Guerra, 2005).

Pode-se afirmar, ainda, que o cuidado em saúde mental infanto-juvenil integra desde questões relacionadas às necessidades básicas da criança e do adolescente, até a criação de estratégias que giram em torno do seu processo formativo. O termo necessidades básicas, utilizado neste artigo, vincula-se à teoria de Winnicott (2000). Segundo esse autor, a preocupação materna primária deve girar em torno da preocupação de a mãe oferecer ao bebê exatamente o cuidado que ele necessita, tanto em termos físicos, quanto emocionais, adaptando-se às necessidades do bebê nos seus momentos iniciais de vida. Assim, as estratégias de cuidado devem ser estruturadas tendo como base as relações iniciais em direção à autonomia.

Ainda objetivando aprofundar as questões referentes às particularidades das estratégias de cuidado da criança e do adolescente com sofrimento psíquico e buscando transpor essa temática para um plano mais amplo, vale ressaltar a necessidade frequente e simultânea do envolvimento de "vários setores autônomos em relação à saúde mental propriamente dita, como os setores da saúde geral/atenção básica, educação, assistência social, justiça e direitos" (Couto et al., 2008, p. 390). Assim, o desafio instituído é a operacionalização, instrumentalização e ampliação das ações de intersetorialidade.

Diante dessa perspectiva, cabe ao CAPSi a atribuição de prestar assistência diária a crianças e adolescentes com sofrimento psíquico severo e persistente, tendo como proposta norteadora: a implementação de ações que visem à reversão da tendência institucionalizante de crianças e adolescentes; a organização da rede existente conforme as diretrizes da política pública de saúde mental; o fortalecimento das redes de apoio comunitárias e familiares; 
a promoção da articulação corresponsável entre os diferentes setores públicos que compõem a rede da criança e do adolescente e a oferta da retaguarda às equipes da Atenção Básica (Brasil, 2005b).

Várias pesquisas demonstram a necessidade de integração entre os serviços de saúde mental (CAPS e CAPSi) e a Atenção Básica ${ }^{1}$ de Saúde (Campos \& Nascimento, 2003; Coimbra \& Kantorski, 2005; Figueiredo \& Campos, 2009). Essas pesquisas sugerem a criação de mecanismos de integração entre esses serviços capazes de favorecer a promoção da vida comunitária e da autonomia dos usuários. Dentro desse cenário, o Apoio Matricial tem se caracterizado como uma das ferramentas que pode proporcionar essa interlocução, já que a sua proposta é dar suporte técnico-pedagógico e retaguarda assistencial para as equipes de referências da Atenção Básica. Entende-se por suporte técnico-pedagógico aquele que produz ação educativa e por retaguarda assistencial aquela que produz ação clínica/terapêutica com os usuários (Campos \& Donitti, 2007).

Nesse cenário, a Rede de Atenção Psicossocial (RAPS), instituída através da Portaria do Ministério da Saúde n 3.088 de dezembro de 2011, surge com o objetivo de favorecer a interlocução entre esses e os demais componentes ${ }^{2}$ de rede de cuidado em saúde mental. Assim, sua implantação visa criar, ampliar e articular pontos de atenção ${ }^{3}$ à saúde para sujeitos com sofrimento psíquico e com necessidades decorrentes do uso abusivo de álcool e outras drogas no âmbito do SUS, impondo algumas mudanças na configuração dos serviços na rede de saúde mental.

ARAPS é uma das redes prioritárias ${ }^{4}$ da Rede de Atenção à Saúde (RAS), que se configura como arranjos organizativos de ações e serviços de saúde, de diferentes níveis de atenção que, integradas por meio de sistemas de apoio técnico, logístico e de gestão, buscam garantir o cuidado integral.
Nesse novo arranjo de rede, a Atenção Básica reafirma sua posição estratégica na produção do cuidado em saúde, tornando-se um dispositivo de comunicação entre os diversos componentes da rede, tendo como atribuições a coordenação do cuidado, a resolutividade e a ordenação da rede (Brasil, 2010).

Mais especificamente no que se refere à atuação da RAPS, seus objetivos visam: a ampliação do acesso à atenção psicossocial da população em geral; a promoção da vinculação dos sujeitos com sofrimento psíquico e com necessidades decorrentes do uso abusivo de álcool e outras drogas e suas famílias aos pontos de atenção; e a garantia da articulação e integração dos pontos de atenção das redes de saúde no território, qualificando o cuidado por meio do acolhimento, do acompanhamento contínuo e da atenção às urgências. Com a implantação desse cenário, os CAPS e os CAPSi são configurados como serviços de atenção psicossocial especializada, responsáveis pela realização de estratégias de desinstitucionalização, vinculando os usuários a uma extensa rede que inclui desde a Atenção Básica, até a urgência e emergência.

No que se refere às mudanças ocorridas no âmbito da saúde mental infanto-juvenil, com a implantação da RAPS, além das transformações já citadas acimas, são realizadas mudanças também nas modalidades de atendimento do CAPSi, com a inclusão da demanda de atendimento de pessoas que fazem uso abusivo do álcool e outras drogas, nesse serviço. No entanto, cabe ressaltar que, apesar de já estar em vigor, as mudanças propostas pela Portaria $\mathrm{n}^{\circ}$ 3.088 ainda se encontram em fase de implantação, sendo incipiente as suas influências na rede de saúde mental infanto-juvenil (Brasil, 2011). O objetivo desse trabalho consiste em compreender os significados conferidos pelos profissionais de saúde sobre as atribuições em um Centro de Atenção Psicossocial Infanto-juvenil (CAPSi).

\section{MÉTODO}

O estudo apresenta abordagem de natureza qualitativa. De acordo com Minayo (2010), as pesquisas dessa natureza têm como foco a compreensão dos significados, dos sentidos,

1 A Atenção Básica é o primeiro nível de complexidade de atenção em saúde. Caracteriza-se por um conjunto de ações de saúde no âmbito individual e coletivo, que abrange a promoção e a proteção da saúde, a prevenção de agravos, o diagnóstico, o tratamento e a reabilitação (Brasil, 2012).

2 A RAPS é formada por sete componentes: atenção básica em saúde; atenção psicossocial especializada; atenção de urgência e emergência; atenção residencial de caráter transitório; atenção hospitalar; estratégias de desinstitucionalização e reabilitação psicossocial (Brasil, 2011).

3 Principais pontos de atenção: Unidades Básicas de Saúde (também chamadas de Postos de Saúde); Núcleos de Apoio à Saúde da Família; Consultórios da rua; Centros de Convivência; Centros de Atenção Psicossocial nas suas diferentes modalidades; Atenção de Urgência e Emergência; Unidades de Acolhimento; Serviços de atenção em regime residencial; Leitos de saúde mental, álcool e outras drogas em Hospitais Gerais e Serviços Residenciais Terapêuticos (Brasil, 2011). das intencionalidades e das questões subjetivas inerentes aos atos, às atitudes, às relações e às estruturas sociais.

Participaram do estudo profissionais que atuavam em um CAPSi de uma cidade da Região Sudeste do Brasil. Os dados foram coletados no ano de 2009, que correspondia ao segundo ano de implantação do serviço. Ao total foram quinze participantes: três psicólogos (uma funcionária desse grupo era diretora da instituição), um arteterapeuta, um assistente social, um enfermeiro, quatro técnicos de enfermagem, dois assistentes administrativos, dois auxiliares

4 De acordo com a RAP, as redes prioritárias são: Rede Cegonha, que tem um recorte de atenção à gestante e de atenção à criança até 24 meses; Rede de Atenção às Urgências e Emergências; Rede de Atenção Psicossocial; Rede de Atenção às Doenças e Condições Crônicas; Rede de Cuidado à Pessoa com Deficiência. Todas as redes também são transversalizadas pelos temas: qualificação e educação; informação; regulação; e promoção e vigilância à saúde (Brasil, 2011). 
de serviços gerais e um vigilante. O serviço foi escolhido por ser o único do estado na modalidade CAPSi, importante informar que assim permaneceu até o ano de 2017. Outro fator que justificou a escolha do serviço foi que o mesmo estava no segundo ano de funcionamento.

O instrumento utilizado foi um roteiro para a coleta de dados em entrevista semiestruturada. Nesse tipo de técnica, o pesquisador deve seguir um conjunto de questões abertas e fechadas previamente definidas, contudo, a sequência das questões deve permitir que a entrevista ocorra de forma semelhante a de uma conversa informal (Boni \& Quaresma, 2005).

Os temas abordados na entrevista foram: tempo de trabalho no serviço; perfil do usuário; encaminhamentos realizados para o CAPSi; fluxo de entrada e inserção do usuário no serviço; encaminhamentos e interlocuções do CAPSi para outros serviços/setores; atribuições do CAPSi na rede de saúde mental infanto-juvenil; atribuições do profissional entrevistado e suas concepções sobre o sofrimento psíquico leve, moderado e grave. Cada entrevista teve duração de, aproximadamente, uma hora. Foram realizadas no próprio serviço, agendadas de acordo com a disponibilidade do participante. Todas foram gravadas e cuidadosamente transcritas.

Os dados foram submetidos à técnica de análise de conteúdo proposto por Bardin (2006). A análise de conteúdo consiste em um conjunto de técnicas de análise das comunicações, que utiliza procedimentos sistemáticos para a descrição do conteúdo das mensagens (Bardin, 2006). De acordo com a proposta de Bardin (2006), foram realizadas as seguintes etapas: (1) pré-análise, (2) exploração de material e (3) tratamento dos resultados.

A fase da pré-análise dos dados se constituiu na leitura flutuante da transcrição das entrevistas, sendo recortados trechos das falas que contemplavam o objetivo do estudo. $\mathrm{Na}$ segunda fase, foi realizada a categorização dos dados, que foram organizados com base nos sentidos e significados conferidos pelos profissionais de saúde sobre as atribuições do CAPSi. Após a transformação de dados brutos, foram obtidas cinco categorias. A última etapa configurou-se na fase do tratamento dos resultados, que culminou na análise reflexiva dos dados, tendo como elementos norteadores as categorias encontradas.

Para a apresentação de cada categoria são explicitados extratos das falas dos participantes. As falas são identificadas por meio da letra $\mathrm{E}$ seguida de um número, representando os entrevistados (E-1, E-2, E-3, etc.)

A realização da pesquisa foi aprovada pelo Comitê de Ética em Pesquisa com Seres Humanos (CEP) e pela Secretaria Municipal de Saúde. Todos os participantes assinaram um Termo de Consentimento Livre e Esclarecido, em atendimento à Resolução $\mathrm{n}^{\circ}$ 466/12 do Conselho Nacional de Saúde.

\section{RESULTADOS E DISCUSSÃO}

Após a análise dos dados brutos, foi possível identificar que as concepções dos profissionais de saúde acerca das atribuições do CAPSi, na rede de atenção em saúde mental infanto-juvenil, giram em torno dos seguintes temas: articulador e organizador da rede; apoio matricial; tipos de casos atendidos no CAPSi; estratégias de cuidado e reinserção social como elemento norteador do tratamento. Concomitantemente à apresentação dos resultados, será realizada a discussão dos dados.

\section{Articulador e Organizador da Rede de Serviços de Saúde Mental Infanto-Juvenil}

Essa categoria engloba as falas que apresentam o CAPSi como um articulador e organizador da rede de serviços de saúde mental. Os relatos revelam que essa atividade é uma questão complexa e, por isso, nem sempre alcança os objetivos desejados. Verifica-se, também, que as falas que representam essa categoria se fizeram presentes em quase todas as entrevistas analisadas, sendo a atribuição de articulador e organizador da rede uma das principais atribuições do CAPSi na concepção dos profissionais entrevistados. Cabe ressaltar que a coleta de dados desse trabalho ocorreu antes da implantação da RAPS, assim, nesse período, a função de organizar a rede de saúde mental infanto-juvenil e coordenação do cuidado era atribuição do CAPSi.

... CAPS esse grande organizador, o grande articulador, no que diz respeito a essa política de atenção à criança e adolescente... (E-09).

... o papel principal do CAPSi tem sido o de articular também essa rede. É... eu já falei isso antes. Parece uma coisa muito óbvia pra gente, que pra você construir um caso, você tem que ter informações de diversos setores, mas muitas vezes isso não é feito (E-01).

Vários CAPS no país inteiro, né, uma ideia de... de fechamento, aquilo que a gente chama de enclausuramento ou de encapsulamento, então o grande desafio de um CAPS é ele ser exatamente essa função que ele tem de articulador e organizador de rede ...(E-03).

Conforme citado anteriormente, com a implantação das RAPS (Brasil, 2011) ocorre uma nova configuração na organização e articulação dos serviços e ações de saúde mental na rede de atenção. A Atenção Básica assume o centro da rede, sendo um dos dispositivos responsáveis por ser a porta de entrada preferencial do sistema. Isso ocorre porque esse nível de atenção tem as responsabilidades de reconhecer 
as necessidades de saúde do seu território de abrangência; de organizar as necessidades dessa população em relação aos outros pontos de atenção à saúde; de acompanhar e organizar o fluxo dos usuários entre os pontos de atenção das RAPS, bem como promover ações intersetoriais (Brasil, 2011).

Diante dessa realidade, o CAPSi compõe a RAPS enquanto um dos seus componentes responsáveis também pela ordenação do cuidado e organização da rede em saúde mental infanto-juvenil, no entanto, essa função torna-se complementar às ações desenvolvidas pela Atenção Básica, setor no qual é atualmente atribuído o papel de articulador de rede. Nota-se que a proposição RAPS alinha-se ao Movimento da Reforma Psiquiátrica (Brasil, 2005), no que se refere à produção de cuidado territorializada e à reinserção social. Ou seja, a configuração da Atenção Básica enquanto um dispositivo responsável por acolher e organizar a rede de saúde mental, possibilita a circulação dos usuários pelos serviços da rede de saúde em geral e pelo território, o que contribui, consequentemente, para a sua autonomia e inserção, como também para a redução do estigma da loucura.

Um aspecto que chama a atenção nessa categoria é a palavra encapsulamento, presente em uma das falas. Observa-se que o entrevistado realiza uma construção metafórica, para simbolizar o tensionamento vivenciado no interior do CAPSi na busca de promover a abertura do serviço e não permitir a caracterização do serviço somente como um ambulatório.

Cavalcante et al. (2014) relatam que o termo encapsulado tem sido utilizado para representar a inexistência da relação dos CAPS com outros espaços do território. Nessa forma de funcionamento, a assistência fica restrita ao interior do CAPS, sem o desenvolvimento de ações extramuros e de caráter intersetorial. Os CAPS são caracterizados como serviços substitutivos ao hospital psiquiátrico de base territorial. Assim, suas ações de reabilitação devem utilizar os espaços da própria comunidade, fazendo com que o usuário retome sua posição na sociedade por meio de um processo que envolve construção de autonomia, liberdade e cidadania. Para tanto, reconhecer os recursos territoriais como instrumentos importantes na produção do cuidado, realizar parcerias interinstitucionais, bem como mobilizar e incentivar a criação de espaços que atendam às reais necessidades da criança e do adolescente com sofrimento psíquico, são ações a serem desenvolvidas no CAPSi para evitar o seu encapsulamento.

\section{Apoio Matricial}

A categoria Apoio Matricial apresenta as falas que dizem respeito ao suporte técnico-pedagógico e assistencial, que os profissionais do CAPSi devem prestar às equipes da Atenção Básica dentro do escopo das atribuições do CAPSi, bem como as parcerias que são desenvolvidas entre essas equipes. Na análise dos dados, identifica-se a presença de falas que estão em consonância com a proposta do Ministério da Saúde para a realização do Apoio Matricial:

...fazer o que a gente chama de matriciamento, quer dizer, dar esse apoio aos profissionais de ponta, estar junto com os profissionais discutindo os casos, pra que esses casos que surjam lá não precisem vir aqui, que eles talvez possam ser acompanhado lá, e que se tiver que vir pra cá, venham em última instância, mas uma vez vindo aqui, não quer dizer que a unidade se desresponsabilize do caso que está sendo acompanhado aqui (E-09).

...tá dando suporte matricial às unidades, tá? Parceria, o tempo todo, e... investimento (E-06).

"Estar junto (...) discutindo os casos", "parceria" e "investimentos" fazem parte de um conjunto de atitudes necessárias para o desempenho da prática do Apoio Matricial. Além disso, os dados indicam a ideia do cuidado corresponsável entre as equipes de saúde mental e de Atenção Básica. O Apoio Matricial surge a partir da constatação de que o Movimento da Reforma Psiquiátrica não pode avançar se a Atenção Básica não for incorporada ao processo. No entanto, sabe-se que as equipes de Atenção Básica se sentem desprotegidas para lidar cotidianamente com as demandas de saúde mental. Assim, o matriciamento visa estabelecer relações de parceria entre a saúde mental e a Atenção Básica, oferecendo o suporte técnico-pedagógico a essas equipes através das discussões dos casos identificados no território e da sua corresponsabilização (Dimenstein et al., 2009).

Cabral et al. (2001) colocam a corresponsabilização como uma prática integrada que favorece uma atuação ampla, na qual a equipe ora passa a ser responsável direto pelo cuidado, ora responsável por acompanhar o cuidado. Assim, a corresponsabilização nada mais é que uma postura ética por parte de todos os profissionais envolvidos com um "caso", uma vez que proporciona a conexão de diversos saberes em diferentes níveis de complexidade e, ao mesmo tempo, proporciona que esses profissionais assumam a responsabilidade sobre o cuidado, independentemente do local para que a criança ou o adolescente seja encaminhado, "evitando assim que a família percorra uma verdadeira via crucis até encontrar o serviço mais indicado" (Ribeiro \& Paula, 2013, p. 338), o que Delfini, Bastos e Reis (2017) nomearam de peregrinação familiar.

Para Campos e Domatti (2007), a prática do Apoio Matricial proporciona a ampliação da capacidade resolutiva da Atenção Básica, possibilitando a corresponsabilidade do cuidado de maneira longitudinal. Nessa proposta, os encaminhamentos excessivos dão lugar à resolutividade dos problemas, antes considerados difíceis no território. Além disso, permitem a continuidade do cuidado e o encaminhamento implicado, ou seja, aquele que encaminha deve acompanhar o encaminhamento do "caso". 


\section{Tipos de Casos Atendidos pelo CAPSi}

Essa categoria, diz respeito às principais características das crianças e dos adolescentes acompanhados no CAPSi. De acordo com as falas apresentadas, os usuários desse serviço são caracterizados por possuírem um quadro clínico de sofrimento psíquico severo e persistente. Os participantes da pesquisa entendem que o atendimento desse tipo de caso está entre as atribuições do CAPSi:

CAPS é uma unidade de transtorno mental grave. Quando são transtornos mentais leves, é reencaminhado pra unidade básica. Ou é algum problema neurológico, outros serviços que não têm aqui, a gente tem parceria com APAE, ou com outras instituições, né? (E-11)

Até porque aqui é um centro de especialidade, assim. Então a gente... tem critério, sim, não é qualquer caso. Que a gente vai, né, absorver. Aqui a gente absorve casos com transtornos graves (E-13).

Conforme já afirmado anteriormente, os CAPSi's têm, como uma das suas atribuições, realizar a atenção diária de crianças e adolescentes com comprometimentos psíquicos severos e persistentes. São incluídos, nessa modalidade de atendimento, "os portadores de autismos, psicoses, neuroses graves e todos aqueles casos que, por sua condição psíquica, estão impossibilitados de estabelecer ou manter laços sociais, e que demandam cuidados intensivos" (Brasil, 2004, p.23).

Hoffmann, Santos e Mota (2008), em um estudo realizado para a caracterização do perfil dos usuários do CAPSi, identificaram que os diagnósticos mais frequentes nesse serviço são os transtornos de comportamento. Os diagnósticos de psicose foram mais comuns entre o grupo etário de 15 a 21 anos. Os autores também identificaram a elevada presença de diagnósticos relacionados a problemas de habilidades escolares, o que ressalta a necessidade de uma articulação entre a saúde e a área da educação, com a finalidade de reduzir os encaminhamentos feitos aos CAPSi relacionados a problemas de aprendizado.

$\mathrm{Na}$ análise das falas apresentadas nessa categoria, também foi possível identificar a existência de alguns relatos que reforçam o movimento de medicalização e patologização da infância. A fala do profissional $E$-11 demostra que qualquer comportamento desviante deve ser tratado por um posto de saúde, ou seja, deve ser enquadrado em um diagnóstico e, então, medicado:

Malcriado... Então não tem conduta, é má conduta. Então isso não é pra CAPS isso daí. Isso da minha parte isso teria que ser tratado em posto de saúde (E-11).

Cruz (2010) refere-se à banalização do uso de medicamentos, entendendo esse fenômeno como uma via fácil para minimizar o sofrimento do outro, sendo o medicamento visto como a única forma de alívio do sofrimento para esse sujeito. O autor ressalta ainda que, somado à medicalização, vem a patologização. Nesse cenário, muitas vezes o diagnóstico assume a identidade do sujeito, provocando o fenômeno da manicomialização, em que o indivíduo não está mais enclausurado em uma instituição, mas em seu próprio rótulo.

Quando essa lógica se aplica na infância, retira-se da criança e do adolescente o direito de desenvolver seu papel social para assumir a identidade do louco ou do desviante. $\mathrm{O}$ grande número de crianças diagnosticadas com algum tipo de sofrimento psíquico, bem como a psiquiatrização do discurso familiar, escolar e judiciário, evidencia que há uma tendência social de enquadrar a criança e o adolescente em rótulos, sem uma análise do que essa situação pode provocar no desenvolvimento desses sujeitos.

\section{Estratégias de Cuidado}

A categoria estratégia de cuidado incorpora as falas que dizem respeito às atribuições do CAPSi nas ações que possuem caráter terapêutico. Alguns profissionais relataram as suas estratégias de cuidado tendo como base o referencial teórico da atenção psicossocial, outros apresentaram falas enraizadas pela lógica manicomial:

O CAPS é ter um espaço para essas crianças e pra esses adolescentes se manifestar. (E-01).

Fazer os procedimentos de maior complexidade, mas não, não tem, a gente não tem muita intervenção aqui, com risco... (E-10).

...aqui não é uma escola, é lugar de terapia, e que as crianças vêm para tratamento. (E-02).

A primeira fala apresentada nessa categoria pressupõe um cuidado que tem como base não só o tratamento clínico, mas uma atenção integral e humanizada da assistência, capaz de potencializar manifestações de criatividade e a existência dos sujeitos. Nesse contexto, pode-se afirmar que as discussões, no campo das práticas humanizadas, vão ao encontro da proposta do modelo de atenção psicossocial, pois a terapêutica não se restringe somente ao uso de fármacos e à clínica tradicional. Há mais recursos terapêuticos do que esses, como, por exemplo, a valorização dos diversos saberes, das opiniões dos usuários/familiares, da escuta, do discurso, dos recursos, do território, da educação em saúde e da dimensão singular (Pinto et al., 2011).

Entretanto, verifica-se a existência de dados contraditórios a essa posição, com a caracterização do CAPSi como um lugar para a realização de procedimentos de maior complexidade, de intervenção e de tratamento clínico tradicional. Nas falas que aparecem esses termos, observa-se que as estratégias de cuidado são norteadas por concepções coerentes com o modelo hegemônico, cujo objeto do trabalho é a doença. De acordo com Guljor (2003), a desconstrução 
do modelo hegemônico, paralelamente à construção de uma nova práxis, constitui-se em um desafio para o Movimento da Reforma Psiquiátrica, que implica em um conjunto de intervenções de longo prazo, com caráter técnico, político e cultural.

É interessante observar também que os dados não indicam as particularidades do cuidado em saúde mental infanto-juvenil. Essa situação reforça a ideia da consolidação dos pressupostos do Movimento da Reforma Psiquiátrica voltada para o público adulto, sendo poucas e localizadas as iniciativas e ações no campo da saúde mental infantojuvenil. De acordo com Guerra (2005), quando o foco do cuidado é a criança e o adolescente com sofrimento psíquico, a interface com o setor educacional, médico e jurídico tem sido essencial para a reinterpretação de uma imagem social deficitária atribuída à criança e ao adolescente. A autora ressalta, ainda, a importância de trabalhar com a família, "o luto da criança imaginarizada e a ressignificação do filho real” (p.141), o lúdico e os novos recursos de linguagem no campo da intervenção (Avellar \& Bertollo, 2008).

Outro aspecto relevante é a inclusão efetiva da família no cuidado, ideia corroborada por Monteiro et al. (2012). A presença da família deve ser obrigatória em toda abordagem e na elaboração dos Projetos Terapêuticos Singulares (PTS)5. "Devido ao alto grau de dependência das crianças e mesmo dos adolescentes, eles precisam dos responsáveis para acessar o serviço e para dar continuidade ao tratamento" (Ribeiro \& Paula, 2013, p. 342). Conhecer, entender e valorizar o ponto de vista da família sobre o problema de saúde mental da criança e do adolescente e sua concreta possibilidade de colaboração na execução das estratégias de cuidado é de fundamental importância para a "resolutividade" do caso.

Reitera-se aqui a necessidade do desenvolvimento de estratégias de cuidados baseadas na maternagem, capazes de suprir as necessidades básicas da criança e do adolescente até a busca da autonomia (Winnicott, 2000). Como exemplo, pode-se citar: acalentar, ninar, alimentar, prover uma temperatura adequada, limpar, confortar, consolar, propiciar um ambiente lúdico, favorecer as interações sociais e os processos educativos, além de proporcionar situações que permitam a compreensão do contexto social no qual está inserido.

\section{Reinserção Social como Elemento Norteador do Tratamento}

Essa categoria agrupa as falas que entendem como atribuições do CAPSi o papel de promover a reinserção

5 O PTS é elaborado com base nas necessidades de saúde de cada usuário, não excluindo suas opiniões e seus projetos de vida, proporcionando uma interação entre trabalhador/usuário/família (Pinto et al., 2011). social da criança e do adolescente com sofrimento psíquico. Verifica-se, nos relatos apresentados, que a reinserção social constitui-se como elemento norteador para o tratamento:

... tentar inserir essas crianças nesses outros espaços, né, na escola, na, em alguma atividade que faz, eu acho que o trabalho tem que ser esse, né (E-08).

Fazer essas crianças serem reinseridas ai fora, no, no ambi... no contexto, na sociedade, melhorar as relações na sua família, né, ser um pou... desenvolver a sua autonomia, sua independência (E-09).

Ela não tá inserida em lugar nenhum, e a proposta do CAPS é o quê? É a inclusão social, fazer com que ela faça parte da sociedade, que ela seja vista, que ela seja aceita, que a sociedade às vezes esconde, né? (E-14).

De acordo com os dados apresentados, a criança e o adolescente com sofrimento psíquico devem ser inseridos na sociedade, na escola e no ambiente familiar. Espaços sociais coerentes com as demandas dessa etapa de vida. As falas também apontam para aceitação da criança e do adolescente no seu contexto social, bem como para a importância do desenvolvimento de práticas com o foco na reinserção social. Entretanto, não é possível identificar nos relados como essa prática é realizada, então surgem questionamentos como: Existe algum projeto de reinserção social?; As redes de apoio sociais estão sendo acionadas?; Ações intersetoriais estão sendo desenvolvidas?; Quais ações estão sendo desenvolvidas para a redução do estigma?

Como já relatado anteriormente, a saúde mental infanto-juvenil tem sido negligenciada tanto na construção de políticas públicas, quanto nas reflexões sobre a particularidade do cuidado. Essa situação não é diferente nas discussões sobre a reinserção social desse público. Estudos como os de Leão e Barros (2011) e Paranhos-Passos e Aires (2013) discutem a reinserção social dos usuários de saúde mental adulto, tendo como foco o mercado de trabalho e a geração de renda. Esse contexto, na grande maioria das vezes, não corresponde às necessidades de reinserção social da criança e do adolescente com sofrimento psíquico.

Ronchi e Avellar (2010) indicam a importância de buscar novas formas de atuação no campo da saúde mental infantojuvenil, com base na construção e reconstrução de projetos de vida capazes de dar voz às crianças e aos adolescentes com sofrimento psíquico, para que esses possam circular nos espaços sociais, realizando trocas com a comunidade e com a família. As autoras ressaltam, ainda, que essa é uma tarefa necessária para a efetivação das funções do CAPSi, quais sejam promover saúde e reinserir o usuário no meio social. 


\section{CONSIDERAÇÕES FINAIS}

Este artigo teve como objetivo analisar as concepções de profissionais de saúde sobre as atribuições de um CAPSi. Os resultados indicam que as atribuições conferidas pelos profissionais, em sua maioria, estão em consonância com a proposta do Ministério da Saúde (2004): articulador da rede, oferecer retaguarda à Atenção Básica, prestar assistência a crianças e adolescentes com sofrimento psíquico severo e persistente, promover a reinserção social e desenvolver práticas com o caráter terapêutico.

Entretanto, foi possível identificar na categoria sobre estratégias de cuidado que os sentidos conferidos às práticas terapêuticas não são homogêneos. Há uma contradição nos modelos que norteiam essas estratégias. Algumas estratégias de cuidado têm, como base, a atenção psicossocial e outras ainda estão enraizadas pelo modelo hegemônico da saúde. Tal realidade reitera a afirmação de que para a efetivação do Movimento do Reforma Psiquiátrica, torna-se necessário a transformação de saberes e de valores culturais e sociais (Brasil, 2005).

O estudo também indica a importância de compreender melhor as atribuições do CAPSi, principalmente diante do novo arranjo imposto com as RAPS, que coloca a Atenção Básica como responsável, prioritariamente por ser a porta de entrada dos usuários de saúde mental infanto-juvenil, atribuindo a esse nível de atenção a função de articulador da rede (Brasil, 2011). Nessa configuração, o CAPSi realiza tais tarefas somente de forma complementar à Atenção Básica. Assim, a RAPS favorece a inserção do usuário de saúde mental nesse nível de complexidade e proporciona a interlocução com a Atenção Básica, além de possibilitar um acompanhamento de maneira integral e longitudinal, permitindo uma abordagem mais complexa sobre os determinantes sociais do processo saúde-doença.
É importante frisar que uma das limitações deste estudo deve-se ao fato da pesquisa ter sido realizado somente em um CAPSi, no entanto, os resultados encontrados são congruentes com os de outras pesquisas publicadas na área. Além disso, não foi possível aprofundar as discussões sobre as particularidades do cuidado em saúde mental infantojuvenil tendo com foco a obra de Winnicott (2000), em que o autor define a preocupação materna primaria. Fica claro que, diante da complexidade do assunto abordado no artigo, os dados suscitaram discussões cuja riqueza se mostrou inesgotável no limite dessas páginas.

Ademais, pondera-se a dificuldade dos entrevistados em contemplar as particularidades do cuidado da criança e do adolescente nas atribuições do CAPSi, reafirmando a necessidade de consolidar e de operacionalizar as políticas públicas em saúde mental infanto-juvenil. É preciso promover uma ruptura com a extensão das práticas destinadas ao público adulto e promover novas estratégias de cuidado capazes de responder às demandas apresentadas pela criança e pelo adolescente. O Projeto Terapêutico Singular (PTS) (Pinto et al., 2011) pode ser um aliado para essas questões, pois tem a singularidade como elemento central.

No entanto, para a efetivação dessa proposta é preciso a construção e implantação de PTSs capazes: de romper com a desinformação técnico-político; de desenvolver ações de intersetorialidade coerentes com essa etapa de vida; de promover o resgate do direito da criança e do adolescente como cidadãos; de dar voz a esse público e de incluir a família em seu processo de elaboração, além de inserir o lúdico no campo das intervenções. Nesse contexto, pode-se afirmar que o desafio posto consiste em reescrever a história do cuidado voltada para esse público a partir de novos princípios éticos e políticos (Guerra, 2005; Avellar \& Bertollo, 2008; Hoffmann, Santos, \& Mota, 2008).

\section{REFERÊNCIAS}

Amstalden, A., Hoffmann, A., \& Monteiro, T. (2010). A política de saúde mental infanto-juvenil: Seus percursos e desafios. In E. Lauridsen-Ribeiro \& O. Y. Tanaka (Orgs), Atenção em saúde mental para crianças e adolescentes no SUS (pp. 33-45). São Paulo: Hucitec.

Amarante, P.D.C. (1995). Loucos pela vida: A trajetória da Reforma Psiquiátrica no Brasil. Rio de Janeiro: Fiocruz.

Avellar, L. Z., \& Bertollo, M. (2008) A saúde mental na infância e adolescência e o diálogo necessário entre as dimensões clínica, ética e política. In E. M. Rosa, L. Souza, \& L. Z. Avellar (Orgs.), Psicologia Social: Temas em debate (pp. 68-87). Vitória: UFES - ABRAPSO: GM Editora.

Bardin, L. (2006). Análise de conteúdo (L. de A. Rego \& A. Pinheiro, Trads.). Lisboa: Edições 70.

Boni, V., \& Quaresma, S. J. (2005). Aprendendo a entrevistar: Como fazer entrevistas em Ciências Sociais. Em Tese, 2(1), 68-80.

Brasil. (2003). Saúde Mental e Atenção Básica - O vínculo e o diálogo necessários. Brasília: Ministério da Saúde.
Brasil. (2004). Saúde mental no SUS: Os centros de atenção psicossocial. Brasília: Ministério da Saúde.

Brasil. (2005a). Reforma psiquiátrica e política de saúde mental no Brasil. Brasília, DF: OPAS.

Brasil. (2005b). Caminhos para uma politica de saúde mental infanto-juvenil. Brasília: Ministério da Saúde.

Brasil. (2010). Portaria $n^{\circ} 4.279$, de 30 de dezembro de 2010. Estabelece diretrizes para a organização da Rede de Atenção à Saúde no âmbito do Sistema Único de Saúde (SUS). Brasília: Ministério da Saúde.

Brasil. (2011). Portaria $n^{\circ} 3.088$, de 23 de dezembro de 2011. Institui a Rede de Atenção Psicossocial para pessoas com sofrimento ou transtorno mental e com necessidades decorrentes do uso de crack, álcool e outras drogas, no âmbito do Sistema Único de Saúde. Brasília: Ministério da Saúde.

Brasil. (2012). Política Nacional de Atenção Básica. Brasília: Ministério da Saúde. 
Brasil. (2012). Resolução $n^{\circ}$ 466, de 12 de dezembro de 2012. Brasília: Conselho Nacional de Saúde.

Brasil. (2013). Atenção psicossocial a crianças e adolescentes no SUS: Tecendo redes para garantir direitos. Brasília: Ministério da Saúde.

Birman, J., \& Costa, J. (1994). Organização de instituições para uma psiquiatria comunitária. In P. Amarante (Org.), Psiquiatria social e reforma psiquiátrica (pp. 41-72). Rio de Janeiro: Fiocruz.

Cabral, B., Lucena, M., Oliveira, M., Gouveia, M., Freitas, P., Pereira, S., \& Câmara, S. (2001) Estação Comunidade. In A. Lancetti (Org.), Saúde Loucura 7. Saúde mental e saúde da família (pp. 109-116). São Paulo: Hucitec,

Campos, F.C.B., \& Nascimento, S.P. (2003). Apoio matricial: Reciclando a saúde mental na atenção básica. Campinas: Mimeo.

Campos, G. W. S., \& Domitt, A. C. (2007). Apoio matricial e equipe de referência: Uma metodologia para gestão de trabalho interdisciplinar. Caderno Saúde Pública, 23(2), 399-407.

Cavalcante, A. C. G., Esperidão, E., Silva N. S., Silva K. S., \& Souza, A. C. S. (2014). Indicativos do processo de avaliação de serviços de Saúde Mental. Revista Eletrônica de Enfermagem, 16(1), 109-116.

Coimbra, V., \& Kantorski, L. (2005) O acolhimento em Centro de Atenção Psicossocial. Revista de Enfermagem, 13, 57-62.

Couto, M. C. V., Duarte, C. S., \& Delgado, P. G. G. (2008). A saúde mental infantil na Saúde Pública brasileira: Situação atual e desafios. Revista Brasileira de Psiquiatria, 30(4), 390-398.

Couto, M. C. V., Duarte, C. S., \& Delgado, P. G. G. (2015). Crianças e adolescentes na agenda política da saúde mental brasileira: Inclusão tardia, desafios atuais. Psicologia Clínica, 27(1), 17-40.

Cruz, M. A. S. (2010). Desafios da clínica contemporânea: Novas formas de "manicomialização". In Conselho Regional de Psicologia, Grupo Interinstitucional Queixa Escolar (Org.), Medicalização de Crianças e Adolescentes (pp. 17-25). São Paulo: Casa do Psicólogo.

Delfini, P.S.S., Bastos, I., Reis, A.A. (2017). Peregrinação familiar: A busca por cuidado em saúde mental infantil. Cadernos de Saúde Pública, 33(12), 2-13.

Dimenstein, M., Severo, A. K., Brito, M., Pimenta, A. L., Medeiros, V., \& Bezerra, E. (2009). O Apoio Matricial em Unidades de Saúde da Família: Experimentando inovações em saúde mental. Saúde e Sociedade, 18(1), 63-74.

Figueiredo, M. D., \& Campos, R. O. (2009). Saúde Mental na Atenção Básica à Saúde de Campinas, SP: Uma rede ou um emaranhado? Ciência e Saúde Coletiva, 14(1), 129-138.

Gonçalves, A.M., \& Sena, R.R. (2001). A reforma psiquiátrica no Brasil: Contextualização e reflexos sobre o cuidado com o doente mental na família. Revista Latino-Americana de Enfermagem, 9(2), 48-55.

Guerra, A. M. C. (2005). A psicanálise no campo da saúde mental infanto-juvenil. Psyché, 9(15), 139-154.

Guljor, A. P. F. (2003). Os Centros de Atenção Psicossocial: Um estudo sobre a transformação do modelo assistencial em saúde mental (Dissertação de Mestrado). Escola Nacional de Saúde Pública da Fundação Oswaldo Cruz, Rio de Janeiro, RJ, Brasil.
Hoffmann, M. C., Santos, D. N., \& Mota, E. L. (2008). Caracterização dos usuários e dos serviços prestados por Centros de Atenção Psicossocial Infanto-Juvenil. Caderno de Saúde Pública, 24(3), 633-642.

Jorge, M. A. S., Alencar, P. S. S., Belmonte, P. R., \& Reis, V. L. M. (2003). O indivíduo e o seu contexto social. In Escola Politécnica de Saúde Joaquim Venâncio (Org.), Textos de apoio em saúde mental: Série trabalho e formação em saúde (pp. 51-71). Rio de Janeiro: FIOCRUZ.

Leão, A., \& Barros, S. (2011). Inclusão e exclusão social: As representações sociais dos profissionais de saúde mental. Interface-Comunicação, Saúde e Educação, 15(36), 137-152.

Monteiro, A., Teixeira, L., Silva, R., Rabelo, K., Tavares, S., \& Távora, R. (2012). Sofrimento psíquico em crianças e adolescentes: a busca pelo tratamento. Revista Escola Anna Nery de Enfermagem, 16(3), 523-529.

Minayo, M.C.S. (2010). O desafio do conhecimento: Pesquisa qualitativa em saúde (12 $\left.{ }^{\mathrm{a}} \mathrm{ed}\right)$. São Paulo: Hucitec.

Observatório Brasileiro de Informações sobre Drogas. (2007). Reinserção social: Definição. Brasília. Recuperado de http:// www.obid.senad.gov.br/portais/OBID/conteudo/index. php?id_conteudo $=11437 \&$ rastro $=$ TRATAMENTO $\% 2$ FRein ser\%C3\%A7\%C3\%A3o+Social/Defini $\%$ C3\%A7\%C3\%A3o

Organização Mundial de Saúde. (2005). Atlas: Child and adolescent mental health resources. Geneva: Autor.

Paranhos-Passos, F., \& Aires, S. (2013). Reinserção social de portadores de sofrimento psíquico: o olhar de usuários de um Centro de Atenção Psicossocial. Physis Revista de Saúde Coletiva, 23(1), 13-31.

Pinto, D. M., Jorge, M. S. B., Pinto, A. G. A., Vasconcelos, M. G. F., Cavalcante, C. M., Flores, A.Z.T., \& Andrade, A. S. (2011). Projeto terapêutico singular na produção do cuidado integral: Uma construção coletiva. Texto Contexto Enfermagem, 20(3), 493-302.

Ronchi, J. P., \& Avellar, L. Z. (2010). Saúde mental da criança e do adolescente: A experiência do Capsi da cidade de Vitória-ES. Psicologia: Teoria e Prática, 12(1), 71-84.

Yasui, S., \& Costa-Rosa, A. (2008). A Estratégia Atenção Psicossocial: Desafio na prática dos novos dispositivos de Saúde Mental. Saúde em Debate, 32, 27-37.

Winnicott, D. W. (2000). A preocupação materna primária. In Da pediatria à psicanálise (pp. 399-405, D. L. Bogomeletz, trad.). Rio de Janeiro, RJ: Imago. (Trabalho original de 1956)

Ribeiro, L. E., \& Paula, S. C. (2013). Política de saúde mental para crianças e adolescentes. In M. D. Mateus (Org.), Políticas de saúde mental: Baseado no curso Políticas Públicas de Saúde Mental, do CAPS Luiz R. Cerqueira. São Paulo: Instituto de Saúde. 